\title{
Erratum to: Modern Business Ethics Research: Concept, Theory and Relationships
}

Hsing-Chau Tseng
Chi-Hsiang Duan
Hui-Lien Tung
Hsiang-Jui Kung

\section{Erratum to: J Bus Ethics \\ DOI $10.1007 /$ s10551-009-0133-x}

The Editor-in-Chief and Springer wish to inform readers of this journal that, in accordance with Springer's Policy on Publishing Integrity, the paper by Hsing-Chau Tseng, Chi-Hsiang Duan, Hui-Lien Tung and HsiangJui Kung published for the Journal of Business Ethics entitled "Modern Business Ethics Research: Concept, Theory and Relationships" has been retracted because it contained material previously published by others without appropriate acknowledgement.

Alex C. Michalos

Editor-in-Chief, Faculty of Arts, Brandon University, Brandon, Manitoba, Canada 